\title{
The Specific Frequency of Globular Clusters in Galaxies.
}

\author{
Bruce Elmegreen \\ IBM Research Division, T.J. Watson Research Center \\ PO Box 218, Yorktown Hts. NY 10598 USA
}

September 17, 1999

\begin{abstract}
Variations in the number of globular clusters per unit galaxy luminosity, which is the specific frequency, and the mass of globular clusters per unit total available mass, are reviewed. Correlations with galaxy luminosity and morphology, and with the properties of the associated galactic clusters are discussed, as are the color distributions of globular cluster populations. Several models for the origin of these variations are summarized, including the tidal stripping and interaction/starburst models. Most of the variations in the specific frequency for elliptical galaxies result from variations in the ratio of total mass to light with a nearly constant efficiency for conversion of gas into globular cluster mass. Smaller additional variations may come from tidal stripping, particularly in $\mathrm{cD}$ galaxies.
\end{abstract}

Keywords: globular clusters, galaxy formation, galactic clusters, galaxy interactions

to be published in "Toward a New Millennium in Galaxy Morphology" edited by D.L. Block, I. Puerari, A. Stockton and D. Ferreira (Kluwer, Dordrecht), 1999, from a conference in Johannesburg, South Africa, September 13-18, 1999

\section{Introduction}

Globular clusters (GC) were among the first objects to form in the Universe. Their distributions relative to galaxies and clusters of galaxies, their elemental abundances, motions, and internal properties like radius and mass, all contribute to our understanding of the origin of structure and the associated early star formation.

One of the fundamental parameters of globular cluster research has been the specific frequency (Harris and van den Bergh 1981)

$$
S_{N}=N_{G C} 10^{0.4\left(M_{V}+15\right)},
$$

which is the number of globular clusters, $N_{G C}$, per unit galaxy luminosity, normalized to a galaxy with an absolute $\mathrm{V}$ magnitude of -15. Spiral galaxies typically have $S_{N} \leq 1$ (van den Bergh and Harris 1982), while dwarf ellipticals (Durrell et al. 1996; Miller et al. 1998), ellipticals (Harris and van den Bergh 1981), and S0 galaxies (Kundu and Whitmore 1998; Chapelon et al. 1999) have $S_{N}$ in the range from 2 to 6 , with lower values in more isolated regions (see review in Harris 
1991). Giant $\mathrm{cD}$ galaxies near the centers of large clusters have larger $S_{N} \sim 10-20$ (Harris and van den Bergh 1981; Harris, Pritchet and McClure 1995; Blakeslee and Tonry 1995; Bridges et al. 1996), with some exceptions (e.g., Kaisler et al. 1996).

This variation of $S_{N}$ from galaxy to galaxy, particularly along morphological classes, has been a long-standing problem in globular cluster research. It seems appropriate at a morphology conference like this to review the nature of this problem and some of the solutions that have been suggested. Unfortunately, there is no completely satisfactory explanation that accounts for all of the available information about globular cluster distributions and properties, but perhaps some combination of the suggested solutions is appropriate. In any case, the observations tell us a lot about the formation of globular clusters, galaxies, and clusters of galaxies.

\section{Properties of globular clusters related to the Specific Frequency}

\section{1. $S_{N}$ Correlations with Galaxy Luminosity}

\subsubsection{Observations}

Djorgovski and Santiago (1992) found that $S_{N}$ increases with the luminosity $L$ of the host galaxy, meaning that the actual number of GC's increases faster than the first power of $L$. Zepf, Geisler, and Ashman (1994) confirmed this using data from the literature, after first noting that a bright non-cluster elliptical, NGC 3923, has a moderately high $S_{N}$.

The question of whether the increase in $S_{N}$ with $L$ is continuous or step-wise was investigated by Kissler-Patig (1997), who distinguished between faint and bright systems: Faint ellipticals are disky with unresolved cores, and they have low $S_{N}$ and low metallicities. Bright ellipticals are boxy with resolved cores, and they have high $S_{N}>5$ and bimodal distributions of GC colors (Sect. 3).

At low galaxy luminosity, particularly among nucleated dwarf ellipticals, the specific frequency decreases with increasing $L$, which is opposite from the case for high $L$ galaxies; non-nucleated dE's have no $L$ trend at all (Miller et al. 1998). However, the average $S_{N}$ for dE's is about the same as for bright ellipticals (Durrell et al. 1996), so the decrease in $S_{N}$ with decreasing $L$ for normal ellipticals turns around at low enough $L$ for the dwarf ellipticals. 


\subsubsection{Theory}

The origin of the $S_{N}(L)$ relation has had various explanations. Aside from the extremely large values of $S_{N}$ for cD galaxies, some of the $S_{N}$ variations among ellipticals is from variations in the mass-to-light ratio, which is known to increase with $L$ (Faber et al. 1987; van der Marel 1991; Pahre et al. 1995).

Kissler-Patig et al. (1997) note that the number of globular clusters in spiral galaxies per unit luminosity of the spheroid, rather than the whole galaxy, is about the same as the number per unit total luminosity for the ellipticals. They suggest that it makes more sense to compare the spheroids anyway because the disks of spirals are still bright with active star formation.

The decrease in $S_{N}$ with increasing $L$ for $\mathrm{dE}$ galaxies has been explained as a result of gas removal during a starburst phase in the main galaxy, after the globular clusters formed (Durrell et al. 1996). Gas removal lowers the mass and luminosity of the galaxy today, but does not change the number of globular clusters. Thus the number per unit luminosity increases with time. If gas removal is more important for lower mass systems, then this increase in $S_{N}$ from birth would be larger for them, explaining the observed correlation.

McLaughlin (1999) explains the whole $S_{N}(L)$ correlation from dE's to giant ellipticals, including cD's, as a combination of effects that operate together while the number of globular clusters $N_{G C}$ per unit total mass remains constant. These effects are: (1) an increase in the star mass-to-light ratio with $L$, noted above; (2) an increase in the x-ray gas mass with $L$, and (3) the selective self-destruction of low mass $\mathrm{dE}$ galaxies, discussed above. Items (1) and (2) give the observed increase in $S_{N}(L)$ for elliptical galaxies at constant $N_{G C}$ /total mass. Item (3) considers the same initial ratio of the number of clusters to the total mass.

The step-wise distinction made by Kissler-Patig (1997) was similarly explained by McLaughlin (1999) after noting that boxy ellipticals have higher x-ray emitting masses than disky ellipticals (Bender et al. 1989). If specific frequency is measured per unit total mass, rather than V-band luminosity, then the two-step distinction of globular cluster populations vanishes.

One of the most compelling early explanations for the increase in $S_{N}$ with $L$ and the variation with Hubble type is that spiral galaxies combine to make ellipticals, and the resulting starbursts during the mergers make large numbers of globular clusters (Ashman and Zepf 1992). There are several problems with the details of this scenario, as discussed in Section 2.2.2, but some elliptical galaxies probably do form by interactions (Toomre 1977, and see below), and globular clusters 
do form in great quantities in interacting galaxies, possibly with large enough numbers to increase $S_{N}$ from the spiral-galaxy value to a low elliptical galaxy value (Schweizer et al. 1996). Interaction models are also interesting because of their prediction of bimodal color distributions for GC populations (Sect. 3). However, most studies of GC populations in old systems discount the standard interaction scenario in which spirals commonly merge to make ellipticals while increasing $S_{N}$. This will be discussed in more detail below.

Another explanation for the $S_{N}(L)$ correlation considers the continuous destruction of GC systems long after they form. Murali and Weinberg (1997) reproduced the observed $S_{N}(L)$ with a variable destruction rate following a uniform $S_{N}$ at birth. The destruction rate varies with the luminosity of the host because the density of elliptical galaxies varies with $L$. Higher density ellipticals, which have lower $L$, destroy their globular clusters faster, giving these ellipticals lower $S_{N}$ today. Murali and Weinberg (1997) also predicted that the mass at the peak of the GC luminosity function should be larger for more completely destroyed GC systems, i.e., for lower mass, lower $S_{N}$, ellipticals. This is because most GC destruction processes, particularly evaporation by 2-body encounters, operate faster for lower cluster masses.

However, the GC luminosity function is not observed to change much from galaxy to galaxy (e.g., Blakeslee, Tonry and Metzger 1997; Kundu and Whitmore 1998), or with position in a galaxy or cluster of galaxies (Harris and Pudritz 1994; Harris, Harris and McLaughlin 1998; Kundu et al. 1999), so the effects of such destruction would have to be confined to unobservably low GC masses, making it inconsequential for $S_{N}$. Nevertheless, the issue of GC destruction can be as important as GC formation in producing $S_{N}$ and other GC correlations, as in the classic explanation for the GC luminosity function by Fall and Rees (1977). More studies of the disruption processes are clearly warranted.

\section{2. $S_{N}$ Correlations with Hubble Type and Morphology}

There are two obvious correlations between $S_{N}$ and galaxy morphology: a sharp increase in $S_{N}$, by a factor of $\sim 6$, going from spirals where $S_{N} \leq 1$ to S0 and elliptical galaxies (van den Bergh 1984; van den Bergh 1995a), and another sharp increase, by about a factor of $\sim 5$, going from bright cluster ellipticals to $\mathrm{cD}$ galaxies.

The spiral value is low partly because spirals still have a lot of star formation. If the spiral luminosity is corrected for fading and extinction, then $S_{N}$ comes to within a factor of $\sim 3$ of the value for ellipticals (van den Bergh 1995a). Or, if the spiral value is measured in terms of the 
mass of the spheroidal component only, then it becomes comparable to the elliptical value (Kissler-Patig et al. 1997).

The second jump to high $S_{N}$ for cD galaxies has had several explanations. Most of these would seem to be of historical interest only because McLaughlin (1999) found that $\mathrm{cD}$ galaxies have normal globular cluster numbers per unit total mass when the x-ray emitting gas mass is considered. Thus the oddity about $\mathrm{cD}$ galaxies may be only that they have three times more optically hidden mass than other ellipticals in the form of dark matter (high $M / L$ values) and x-ray gas. In what follows we review two additional models that have been proposed to increase $S_{N}$ : tidal stripping of globular clusters from smaller galaxies, and interaction-induced globular cluster formation.

\subsubsection{Tidal Stripping Model for Excess Globular Cluster Counts in $c D$ Galaxies}

There are several interesting explanations for the excess GC count in $\mathrm{cD}$ galaxies. One of the most compelling is the stripping scenario. Introduced by Forte et al. (1982) after a suggestion by van den Bergh (1977), and simulated in some detail by Muzzio (1987) and Cote et al. (1998), this model proposes that giant $\mathrm{cD}$ galaxies in the centers of rich clusters have selectively stripped globular clusters from the outskirts of neighboring galaxies, thereby increasing $S_{N}$ for the $\mathrm{cD}$ and decreasing it for the neighbors.

Detailed observations of the supposedly stripped neighbors of some cD galaxies make this scenario plausible. Forbes et al. (1997) suggested that if NGC 4486B near the cD galaxy M87 in Virgo originally had a luminosity given by its velocity dispersion, and if it had a metallicity given by this original luminosity, using the standard correlations, then this companion galaxy must have lost $95 \%$ of its stars since birth and had a metallicity in the middle of the range for the metallicities of the globular clusters in M87. In terms of numbers of GC transferred, 95\% of the original GC count for NGC 4486B would have been $\sim 1000$ GC, and this is one-fifth of the total in M87 that have the same metallicity.

The same calculation can be done for NGC 1404, near the cD NGC 1399 in Fornax (Forbes et al. 1997). If the observed number of GC in NGC 1404 today is subtracted from the expected number of GC at birth, given an initial ratio $S_{N}=5$, then about one-third of the GC in NGC 1399 at the same metallicity could have been transferred from NGC 1404. However, NGC 1404 has a luminosity today that is the same as what would be expected from its velocity dispersion. This implies that NGC 1404 could not have lost a large fraction of its stars. Yet the difference in GC counts from a fiducial value with $S_{N}=5$ represents a $50 \%$ loss. Perhaps the transferred GC were in the outer parts of NGC 
1404, so only the GC went without any accompanying stars. In any case, the tidal radius expected for NGC 1404 is about the same as the observed radius (Forbes et al. 1997), so it looks tidally truncated. NGC 1404 is also located in the x-ray halo of NGC 1399.

Also for NGC 5846A near the larger galaxy NGC 5846 (Forbes et al. 1997), the original metallicity of the smaller galaxy matches the metal poor component of the GC population in NGC 5846, and the number of GC's in the original NGC 5846A $(\sim 800)$ matches the current number at this metallicity in NGC 5846.

Neilsen et al. (1997) made a similar study of NGC 4478, another companion to M87. The GC population in NGC 4478 has only one metallicity value, and this is the same as for the metal-poor population in the two-component color distribution in M87. NGC 4478 also has very few GC today, giving it $S_{N}=0.6$ instead of the usual $S_{N} \sim 5$ for this type of galaxy. As a result, it might have lost $\sim 300$ clusters. NGC 4478 is also so close to M87 that it is currently projected inside the extended GC halo of the $\mathrm{cD}$ galaxy. Thus $\sim 300$ of the blue $\mathrm{GC}$ in M87 could have come from NGC 4478.

Also in support of the stripping model is that the velocity dispersion of the GC system around the cD galaxy NGC 1399 is more similar to the high velocity dispersion of the whole galactic cluster than the smaller dispersion of NGC 1399 itself (Grillmair et al. 1994; Minniti et al 1998; Kissler-Patig et al. 1999). The same is true for cD M87 (Mould et al. 1987; see Sect. 4). Thus many of the GC's in NGC 1399 and M87 could have had an origin outside the galactic boundary. The specific frequency for ellipticals in general also seems to correlate better with the galactic cluster velocity dispersion than with the host galaxy dispersion (Blakeslee et al. 1997; Harris et al. 1998; Kissler-Patig et al. 1999). Specific frequency correlates with local galaxy density as well (Kumai et al. 1993a; West 1993; Blakeslee 1997; Blakeslee et al. 1997).

In addition, the distribution of GC density with galactocentric distance is steeper in the companion galaxies that are supposedly stripped than in the $\mathrm{cD}$, which received the stripped GC (Forbes et al. 1997), so the stripped galaxies look tidally truncated in their GC distributions. Furthermore, the distribution of GC's in presumably unstripped dE galaxies is more extended than the galaxy light (Minniti et al. 1996), so preferential stripping of the GC's alone, which is needed to increase $S_{N}$ in the central galaxy, would seem to be possible.

The problem with stripping models is that they are not likely to increase $S_{N}$ by the necessary amount, which is a factor of $\sim 3$, to explain the high values of this ratio in $\mathrm{cD}$ galaxies (West 1993; Harris et al. 1998). The accumulation of dwarf galaxies by a $\mathrm{cD}$ will not increase $S_{N}$ much either because dwarfs have relatively low $S_{N} \sim 4-6$ themselves 
(Durrell et al. 1996; Minniti et al. 1996). Even if the extended GC envelopes of $\mathrm{dE}$ galaxies were alone stripped to make a high $S_{N}$ in a cD galaxy, the remaining $\mathrm{dE}$ cores are not obviously present nearby (Harris et al. 1998).

Hilker et al. (1999) point out, however, that if the lowest mass dwarfs have values of $S_{N}$ as high as the Fornax and Sagittarius dwarf spheroidals $\left(S_{N} \sim 20-30\right)$, or, at least, if half the dwarfs with magnitudes fainter than -12.5 have one or more GC's, and if the residual gas in the dwarfs also makes GC's as in interacting starburst systems, then dwarf galaxy accretion by $\mathrm{cD}$ galaxies can provide the right number of globular clusters to account for an $S_{N} \sim 10$. In addition, they show that the surface density of dwarf galaxies drops below the extrapolated lower-law distribution toward the center of the Fornax cluster of galaxies, at the same place where the blue globular clusters and $\mathrm{cD}$ halo stars around NGC 1399 have an overabundance.

\subsubsection{Interaction Model for the excess $S_{N}$ in Ellipticals}

The difference in $S_{N}$ between spiral and elliptical galaxies, which is a factor of $\sim 3-6$ depending on how it is counted, was long used as an argument against the model in which ellipticals formed from the merger of spirals (van den Bergh 1984). Then Schweizer (1987) and Ashman and Zepf (1992) suggested that interactions in the early Universe between gaseous spirals made new GC's, thereby increasing $S_{N}$ to the required value by the time the merged remnants looked like ellipticals. Ashman and Zepf predicted that ellipticals should have two populations of GC's, one metal poor from the original spiral GC population, and another metal rich, from the more recent epoch of GC formation during the merger. Remarkably, such bimodal GC populations were soon found (Zepf and Ashman 1993), as discussed in more detail in section 3.

The merger model for the formation of GC's was also confirmed directly by the observation of profuse GC formation during recent gassy mergers, some of which also end up looking like elliptical galaxies (Schweizer 1987; Schweizer et al. 1996; Miller et al. 1997). Starbursts in general make a lot of GC-like objects (Holtzman et al. 1992; Whitmore et al. 1993; Whitmore and Schweizer 1995; see review in Ho 1997).

Other predictions of the merger model have not faired so well, however, and it is unclear today how much mergers played a role in determining the range of $S_{N}$.

One early problem was that mergers were expected to form a lot of stars that are not in globular clusters, so it was unclear why the merger starburst should have the required high $S_{N}$ by itself, and not a "normal $S_{N}$ " from normal star formation (West 1993). This problem may have gone away, however, because starburst mergers do seem to 
put an unusually high fraction of their young stars into GC's rather than unbound associations (Meurer et al. 1995; Schweizer et al. 1996).

Another problem is that S0 galaxies have the same high $S_{N}$ as elliptical galaxies, and no one expects $\mathrm{S} 0$ galaxies to have been made by major mergers (West 1993).

Also, the lowest metallicities for GC systems in elliptical galaxies are higher than the normal metallicities of GC's in spiral galaxies, so the old population of GC's in ellipticals does not look like a GC population from a former spiral (Geisler et al. 1996).

Forbes et al. (1997) listed several other arguments against the merger scenario: (1) metal-poor GC's in elliptical galaxies are usually more numerous than metal-rich GC's, and high $S_{N}$ galaxies have more metalpoor GC's, so the excess in $S_{N}$ cannot be from the recent formation of GC's at high metallicity in a merger. (2) High $S_{N}$ galaxies do not have their metal-rich GC's concentrated in the center where the old starburst should have occurred; rather they have their metal-rich GC's distributed throughout the galaxy, with a large core radius. (There is a slight red GC concentration, however.) (3) A typical cD galaxy needs to have acquired $\sim 10 \mathrm{~L}^{*}$ spirals to get its large mass, and these were likely to have entered at different times in the past, producing different metallicity values for the young GC components; however, cD's generally have only one metal rich component of GC's; (4) some elliptical galaxies with the same luminosity have very different $S_{N}$, so the mergers would have had to produce GC's with different efficiencies; and (5) the main, centralized part of a $\mathrm{cD}$ galaxy is kinematically distinct from its extended envelope, such that the velocity dispersion of the envelope is more like the value in the whole galaxy cluster than the value in the $\mathrm{cD}$ core; in the merger scenario, both the core and the envelope should have been formed by the same mergers.

Kissler-Patig et al. (1998a) and Neilsen and Tsvetanov (1999) seconded the point (1) above. In addition, the metal rich component of GC populations has a metal abundance that scales with the host galaxy luminosity, suggesting it is primordial rather than triggered by interactions (Forbes et al. 1997). When there is a high proportion of high-metallicity GC's in a galaxy, the $S_{N}$ ratio tends to be low, so large $S_{N}$ values should be identified with the primordial metal-poor component of GC's, rather than a younger component that is metalrich (Forbes et al. 1997). Harris (1995) and Harris et al. (1998) also argue against the merger model for large $S_{N}$ galaxies, but say that spiral mergers may account for the low values of $S_{N} \sim 2$ in relatively isolated ellipticals.

Geisler et al. (1996) gives a specific example where the merger model seems to fail: the two metallicity peaks in the elliptical galaxies M49 
and M87 have the same values, and the same ratios of high to low metallicity GC's, but $S_{N}$ is much higher in M87 than in M49. Thus, the excess in $S_{N}$ for M87 cannot be related to its young, metal rich population. Also, the giant elliptical/S0 M86 has only the blue GC population (Neilsen and Tsvetanov 1999).

\subsubsection{Summary of $S_{N}$ dependence on Morphology}

After McLaughlin (1999) showed that $S_{N}$ variations with morphology can be explained mostly by variations in the mass-to-light ratio and the amount of x-ray gas, so that the mass ratio of GC mass to total baryon mass is about constant even though the luminosity ratio in the definition of $S_{N}$ is not, there is little compelling reason to look elsewhere for effects that increase $S_{N}$ substantially.

Nevertheless, gas-rich galaxy mergers may have increased $S_{N}$ a little (to $S_{N} \sim 2$ ) in some ellipticals, and $\mathrm{cD}$ galaxies may have acquired large numbers, but perhaps only modest fractions, of their GC's from stripped neighbors.

\section{Bimodal Color Distributions for Globular Cluster Systems}

An important clue to the early history of elliptical galaxies is the color distribution, or equivalently, metallicity distribution of their globular clusters. Because of line blanketing at short wavelengths in metal-rich stars, red old clusters have more metals than blue old clusters.

A remarkable observation is that globular cluster populations in giant elliptical galaxies tend to have two components, red and blue (Harris et al. 1992; Ostrov et al. 1993; Zepf and Ashman 1993; Lee and Geisler 1993; Secker et al. 1995; Zepf, Ashman and Geisler 1995).

Geisler et al. (1996) studied M49, and found that the metal-rich GC component is more concentrated in the center of the galaxy and the GC's are more metal poor than the galaxy at all radii. Geisler et al. also compared the GC populations in 5 other giant ellipticals previously studied and found that the metallicity peaks are always separated by about 1 dex, and that the most metal poor GC system is always more metal rich than the GC's in spiral galaxies. This latter point was also made by Forbes et al. (1996) for 14 elliptical galaxies, although KisslerPatig et al. (1998a) found that the most metal poor GC's in the cD galaxy NGC 1399 have the same metallicity as the halo GC's in the Milky Way.

The metal-rich component of GC's seems to be intimately connected with the host galaxy because the metallicity of this component scales 
with the galaxy luminosity whereas the metallicity of the metal poor component does not (Forbes et al. 1997; Cote et al. 1998). Also, in M49 at least, the metallicity of the metal-rich GC component nearly mimics the metallicity of the galaxy halo (Geisler et al. 1996); in M87, the metal-rich component is only slightly more metal poor than the galaxy (Kundu et al. 1999). In the S0 galaxy NGC 3115, the metal-rich GC component follows the thick disk light distribution, whereas the metal poor component follows the halo (Kundu and Whitmore 1998).

Only the brightest elliptical galaxies, including some cD's, have bimodal color and metallicity distributions for the GC systems (KisslerPatig 1997). Yet surprisingly, the bright elliptical M86 in Virgo, does not; it has only the blue, metal-poor component (Neilsen and Tsvetanov 1999). M86 is also bluer than the other ellipticals as a whole, so presumably what causes the red GC populations in other galaxies also gives their hosts the same high metallicity.

Another oddity is that the red GC radii tend to be $20 \%$ smaller than the blue GC radii in the two galaxies where this has been measured: NGC 3115 (Kundu and Whitmore 1998) and M87 (Kundu et al. 1999).

The bimodal nature of GC systems in most giant elliptical galaxies is not understood. There were clearly several epochs of GC formation involved, but whether they took place inside these galaxies in two bursts of star formation, or outside the galaxies in separate systems that later merged, is not known. The connections between either component, galaxy interactions, and $S_{N}$ are not clear either. For example, Geisler

et al. (1996) noted that M49 and M87 have the same bimodal color distributions for their GC systems, but M87 has much higher $S_{N}$ than M49.

\section{Inter- and Extra-galactic formations of globular clusters}

The GC systems in the outer parts of $\mathrm{cD}$ galaxies, particularly the blue or metal-poor components, tend to correlate in their properties with the whole galactic cluster rather than the galaxy itself.

The various galaxy-cluster correlations are of three types:

- cluster density: $S_{N}$ increases with the local density of galaxies in a cluster (Kumai et al. 1993a; West 1993; Blakeslee 1997; Blakeslee et al. 1997).

- cluster velocity dispersions: the velocity dispersions of GC systems near $\mathrm{cD}$ galaxies are much larger than the dispersions of the galaxy stars and more comparable to the dispersion in the cluster as a whole, often increasing continuously with radius in 
the cluster (Mould et al. 1987; Huchra and Brodie 1987; Mould et al. 1990; Brodie and Huchra 1991; Grillmair et al. 1994; Cohen and Ryzhov 1997; Minniti et al. 1998; Kissler-Patig et al. 1998a, 1999). Also, $S_{N}$ increases with the velocity dispersion of the host galaxy (Kumai et al. 1993a) and with the velocity dispersion of the whole cluster (Blakeslee 1997; Blakeslee et al. 1997; Harris, Harris, and McLaughlin 1998;

- $x$-ray properties: the excess in $S_{N}$ for giant cluster ellipticals over a value of $\sim 4$ increases with the local galactic cluster density, as determined from the x-ray temperature and position in the cluster (West et al. 1995; Blakeslee et al. 1997). Straight correlations with x-ray temperature are also present, but less obvious (Harris, Harris and McLaughlin 1998).

These cluster-wide correlations imply that GC systems in some giant ellipticals and $\mathrm{cD}$ galaxies come from the cluster as a whole, forming either in cluster gas before the individual galaxies condensed, or forming in and around the individual galaxies but migrating to the cluster centers after getting stripped during subsequent encounters. Problems with stripping as a sole mechanism to increase $S_{N}$ in cD galaxies were discussed above. The other option, whole cluster formation, might account for the blue population because that formed first in a cluster, and it tends to be at large radii around the giant ellipticals, but the blue population is at most about half of the total in giant ellipticals, so $S_{N}$ would not change much if the blue GC's alone came from the galaxy cluster.

McLaughlin et al. (1994) and Blakeslee and Tonry (1995) noted that only the least dynamically evolved clusters, with Bautz-Morgan types BM II and III, have cD galaxies with high $S_{N}$ values. Giant, centralized elliptical galaxies in the highly evolved BM I clusters have normal $S_{N}$. McLaughlin et al. (1994) suggested that the more evolved BM I clusters have had time to dilute initially high $S_{N}$ values in their centers with lower $S_{N}$ material from merged and stripped neighbors. The less evolved BM II and III types are still experiencing merging, as shown, for example, by multiple velocity components in the nuclei of some galaxies (Blakeslee and Tonry 1992). This implies that if cD's with large $S_{N}$ get their extra GC's from "partial" stripping, then they have to do this quickly, before they merge more completely with the remaining low- $S_{N}$ galaxies. Blakeslee and Tonry (1995) also showed that only $20 \%$ of the GC's in the Virgo galaxies surrounding M87 would be required to account for the high $S_{N}$ in M87 if they were stripped, and that this same fraction is likely to have been stripped for typical companion distances (based on Merritt 1988). 
Other models for high $S_{N}$ in clusters involve GC formation in cooling flows (Fabian, Nulsen, and Canizares 1984; Fall and Rees 1985). This idea is generally discounted now because there are $\mathrm{cD}$ galaxies with low $S_{N}$ in clusters with high x-ray emissions, as well as $\mathrm{cD}$ galaxies with high $S_{N}$ in clusters with low x-ray emission (West 1993; Harris, Pritchet and McClure 1995; Kaisler et al. 1996).

Another model that accounts for high $S_{N}$ in the centers of dense clusters, as well as the constancy in the globular cluster luminosity function and characteristic mass, is the biased GC formation model by West (1993). In this model, GC's and other stars form only where the total density exceeds some specific value. Since the background density is relatively high in the centers of clusters, a lot of GC's formed there early on, making the blue GC population. The lower-density outskirts of clusters, and the lower-density clusters and galaxy groups, formed relatively few GC because the critical density was harder to reach. There have been relatively few comments on this model in the literature, but it is difficult to rule out considering the popularity of biased galaxy formation in general (Kaiser 1984; Davis et al. 1985; Bardeen et al. 1986). However, there is still some question about what is most constant, the number of GC per unit baryon mass (McLaughlin 1999) or the number of GC per unit total mass (including dark matter; Blakeslee 1997). The biasing model would seem to be most appropriate in the latter case.

An important observation that may shed some light on early star formation and merging in galaxy clusters is the presence of planetary nebulae and diffuse star light between the galaxies. Thuan and Kormendy (1977) found that background light in the Coma cluster is nearly half $(45 \%)$ of the total light coming from the galaxies, and that the color of this light is about the same blue as the outer halo of the central $\mathrm{cD}$, M87. This suggests that the $\mathrm{cD}$ halo and the intergalactic stars were made from the same, presumably stripped, material, and adds support to the idea, mentioned above, that the extended GC system around M87 is from the cluster at large (see also Weil et al. 1997).

Other evidence for intergalactic stars in clusters is the recent observation of planetary nebula in Virgo (Arnaboldi et al. 1996; Mendez et al. 1997) and Fornax (Theuns and Warren 1997), and the observation of red giants in Virgo (Ferguson et al. 1998). These studies also estimate that stray stars amount to an extra mass that is about half the known galactic mass. 


\section{A Constant Globular Cluster Formation Efficiency}

van den Bergh (1994) noted that globular clusters amount to about $2 \%$ of the total luminosities of the halo stars in both the Milky Way and the LMC. This would imply a similar cluster formation efficiency in two different galaxies. Other observations have suggested the same thing. Richer et al. (1993) and Harris, Pritchet and McClure (1995) noted that $\sim 1 \%$ of the gravitating mass in clusters of galaxies turns into globular clusters. Zepf and Ashman (1993) and Harris and Pudritz (1994) similarly pointed out that the GC mass formation efficiency is more constant than the luminosity efficiency, as measured by $S_{N}$. Durrell et al. (1996) suggested that globular clusters represent about $1 \%$ of all star formation in order to get the metal enrichment needed to account for the difference between the GC metals and the associated galaxy metals. If the overall efficiency of star formation in gas is $\sim 10 \%$, then the globular cluster efficiency per unit gas mass would be about $0.1 \%$.

Blakeslee, Tonry and Metzger (1997) used the velocity dispersions of galaxy clusters to get the total cluster masses within $40 \mathrm{kpc}$ (including dark matter), and found that the number of globular clusters per unit cluster mass is about constant, equal to $\sim 0.7$ GC's per $10^{9} \mathrm{M}_{\odot}$ of galaxy cluster. Considering an average GC mass of $10^{6} \mathrm{M}_{\odot}$, they converted this to $<0.1 \%$ (actually 0.0007 ) $\mathrm{M}_{\odot}$ of globular clusters per $M_{\odot}$ of galaxy cluster mass.

Harris, Harris and McLaughlin (1998) suggested that an average of 5.8 globular clusters form in each $10^{9} \mathrm{M}_{\odot}$ of galaxy cluster baryonic mass, so if the average GC mass is $3 \times 10^{5} \mathrm{M}_{\odot}$, which they assumed, then the formation efficiency is $0.0018 \mathrm{M}_{\odot}$ of $\mathrm{GC}$ per $\mathrm{M}_{\odot}$ of total baryon mass. They also suggested that the efficiency is generally lower in more massive galaxy clusters, and that it is larger in denser galaxy clusters, unless bright cluster ellipticals limit their own brightnesses through star-formation induced winds (Blakeslee et al. 1997).

McLaughlin (1999) recovered the constant cluster formation efficiency by considering the x-ray gas in clusters. He got a value of 0.0025 $\mathrm{M}_{\odot}$ of $\mathrm{GC}$ for each $\mathrm{M}_{\odot}$ of primordial gas in a galaxy cluster. This is about the same as the local mass efficiency of bound cluster formation in the Milky Way (Harris and Pudritz 1994).

Observations of a constant efficiency for GC formation leave open a question about the efficiency of non-clustered star formation (Larsen, private communication). If $S_{N}$ varies, then the number of GC per nonclustered star varies, and if x-ray gas mass is needed to make the efficiency of GC formation constant, then there must also be variations in the ratio of GC mass to gas mass that does not form stars. Why is the 
GC formation efficiency more constant than the formation efficiency of other stars? Do GC form first with this constant efficiency, and then destroy the remaining clouds with a variable rate that depends on other properties of the environment?

These studies show that globular clusters form in a more-or-less uniform fashion in galaxy clusters, with an efficiency that is not much different from that of normal open clusters. This probably implies that the star formation processes were not much different in the early Universe than they are today. How all of this relates to the bimodal color distributions of globular clusters, to the likely stripping of neighbors by giant $\mathrm{cD}$ galaxies, and to GC destruction over time, is not clear. The complexity of this problem illustrates that globular clusters today should be viewed in the context of the entire history of the Universe, ranging from star formation and enrichment before most of the galaxies formed, to galaxy evolution and globular cluster destruction over time, to galaxy interactions, cluster stripping, and galaxy cluster evolution.

\section{Summary}

The globular cluster specific frequency, $S_{N}$, is smaller by a factor of $\sim 6$ in spiral galaxies than in ellipticals (or a factor of $\sim 3$ if spiral galaxy fading and extinction are considered), and smaller by another factor of $\sim 5$ in ellipticals compared to giant $\mathrm{cD}$ galaxies in Bautz-Morgan type II and III clusters. Some of this variation is the result of changes in the mass-to-light ratio, particularly among elliptical galaxies with increasing $L$, and some is the result of changes in the total gas mass (seen in x-ray) to the galaxy luminosity. Variations in $S_{N}$ by a factor of $\sim 2$ seem likely for merging galaxies, which make globular clusters during a starburst phase and leave the system looking like an elliptical galaxy.

The efficiency of globular cluster formation per unit total mass, or per unit total gas mass, is remarkably constant in different environments because most of the $S_{N}$ changes are accounted for by changes in the ratio of total mass to luminosity. Moreover, this near-constant mass efficiency is about the same as the efficiency of bound cluster formation in galaxy disks today. This result implies that globular clusters may

have formed by normal star formation processes (Harris and Pudritz 1994; Elmegreen and Efremov 1997).

Much of this globular cluster formation occurred very early in the Universe, before the galaxies were pieced together. This gave the blue population of globular clusters in elliptical galaxies and the halo population in spirals (which have the lowest metal abundances of all). The 
enrichment that resulted from this cluster formation, and from all of the associated non-cluster star formation, provided the metals for the galaxies that were soon to form in the same regions, from the residual and recycled gas.

When the elliptical galaxies formed in this second phase, more globular clusters formed too, along with non-clustered stars, giving the red and galaxy-correlated populations of globulars in the elliptical galaxies, with their larger metal abundances. The analogous second phase of galaxy building for spirals occurred primarily in the disks, where additional, metal-rich globular clusters formed too.

After the globular clusters and galaxies formed, the galaxies began to interact, first stripping off the peripheral clusters that were most loosely bound, and collecting these, along with loosened stars, in the galaxy cluster potential wells. The interactions continued by direct merging of gassy systems in the field. This phase formed the cD's in clusters of galaxies, with their relatively large $S_{N}$ values compared to cluster ellipticals, and it also formed some of the ellipticals in the field, with their relatively low $S_{N}$ values compared to cluster ellipticals.

While this scenario makes a lot of sense, and goes a long way toward explaining $S_{N}$ and other properties of globular cluster systems and their associated galaxies, there are some perplexing issues that have no satisfactory explanations at all. We list them here for future study:

- Why is the globular cluster luminosity function, or mass function, so constant from place to place? Does this constancy imply that it is the same as the initial cluster mass function? If so, then why is there a characteristic mass (when all other "normal" star formation operates in a scale-free interstellar medium). If today's cluster mass function is not the initial function, then how could cluster erosion make today's function the same in all environments?

- Why is the bound cluster formation efficiency so constant from place to place and over time? How much does it vary with total star formation rate (Larsen and Richtler 1999) or other local properties of the environment? If $S_{N}$ varies even when the efficiency of globular cluster formation is constant, does this mean that the efficiency of non-clustered star formation is varying too (S. Larsen, private communication)? Why would the efficiency of GC formation be more constant than the efficiency of all star formation?

- When and how did the red population of globular clusters form in bright elliptical galaxies? Did it form during interactions or in situ? Did any other sub-populations of globular clusters form during interactions or at later times and remain obscure because 
of a conspiracy between age and metallicity (Kissler-Patig et al. 1998b)? Did the blue population of globular clusters in giant ellipticals come from stripped neighbors? If so, why did it get so well distributed throughout the galaxy and not just remain in the outer regions?

- Why does some star formation produce bound clusters and other star formation not? That is, why does the local efficiency of star formation in a cloud core vary (being higher where bound clusters form)? Is the high efficiency the result of compression and triggering, as appears to be the case for many galactic disk clusters today (Elmegreen et al. 1999)? Do the high pressures required for globular cluster formation (Elmegreen and Efremov 1997) also imply some type of triggering, or cloud collisions (Kumai et al. 1993b). If so, then why do globular clusters also form in very low mass galaxies, where the velocity dispersion and total gas pressure are extremely low (van den Bergh 1995b)?

Some of these problems are related to star formation in general, and others to galaxy formation and evolution. We might have to understand all of these aspects of globular clusters together before we can understand any one part of the picture by itself.

\section{Acknowledgements}

Financial support for attending this conference was kindly provided by the Anglo American Chairman's Fund, and SASOL. Helpful comments on the manuscript by Drs. T. Richtler, D. McLaughlin, and S. Larsen are appreciated.

\section{References}

M. Arnaboldi, K. C. Freeman, R. H. Mendez, M. Capaccioli, R. Ciardullo, H. Ford, O. Gerhard, X. Hui, G. H. Jacoby, R. P. Kudritzki, and P. J. Quinn. ApJ, 472:145, 1996.

K. M. Ashman and S. E. Zepf. ApJ, 384:50, 1992.

J. M. Bardeen, J. R. Bond, N. Kaiser, and A. S. Szalay. ApJ, 304:15 1986.

R. Bender, P., Surma, S., Döbereiner, C., Möllenhoff, and R. Madejsky. A\&A, 217:35,1989.

J. P. Blakeslee. ApJ, 481:L59, 1997.

J. P. Blakeslee and J. L. Tonry. AJ, 103:1457, 1992.

J. P. Blakeslee and J. L. Tonry. ApJ, 442:579, 1995.

J. P. Blakeslee, J. L. Tonry, and M. R. Metzger. AJ, 114:482, 1997. 
T. J. Bridges, D., Carter, W. E., Harris, and C. J. Pritchet. MNRAS, 281:1290, 1996.

J. P. Brodie and J. P. Huchra. ApJ, 379:157, 1991.

S. Chapelon, V., Buat, D., Burgarelle, and M. Kissler-Patig. A $\& A$, 346:721, 1999.

J. G. Cohen and A. Ryzhov. ApJ, 486:230, 1997.

P. Cote, R. O. Marzke, and M. J. West. ApJ, 501:554, 1998.

M. Davis, G., Efstathiou, C. S. Frenk, and S. D. M. White. ApJ, 292:371, 1985.

S. Djorgovski and B. X. Santiago. ApJ, 391:L85, 1992.

P. R. Durrell, W. E. Harris, D. Geisler, and R. E. Pudritz. AJ, 112:972, 1996.

B. G. Elmegreen and Y. N. Efremov. ApJ, 480:235, 1997.

B. G. Elmegreen, Y. N. Efremov, R. E. Pudritz, and H. Zinnecker. In V. G. Mannings, A. P. Boss, and S. S. Russell, editors, Protostars and Planets, IV, University of Arizona, Tucson, in press, 1999.

S. M. Faber, A. Dressler, R. L. Davies, D. Burstein, D. Lynden-Bell, R. Terlevich, and G. Wegner. In S. M. Faber, editor, Nearly Normal Galaxies, Springer, New York, p. 175, 1987.

A. C. Fabian, P. E. J. Nulsen, and C. R. Canizares. Nature, 310:733, 1984.

S. M. Fall and M. J. Rees. MNRAS, 181:37p, 1977.

S. M. Fall and M. J. Rees. ApJ, 298:18, 1985.

H. C. Ferguson, N. R. Tanvir, and T. von Hippel. Nature, 391:461, 1998.

D. A. Forbes, M. Franx, G. D. Illingworth, and C. M. Carollo. ApJ, 467:126, 1996.

D. A. Forbes, J. P. Brodie, and C. J. Grillmair. AJ, 113:1652, 1997.

J. C. Forte, R. E. Martinez, and J. C. Muzzio. AJ, 87:1465, 1982.

D. Geisler, M. G. Lee, and E. Kim. AJ, 111:1529, 1996.

C. J. Grillmair, K. C. Freeman, G. V. Bicknell, D. Carter, W. J. Couch, J. SommerLarsen, and K. Taylor. ApJ, 422:L7, 1994.

W. E. Harris 1991. ARAA, 29:543, 1991.

W. E. Harris, In P. C. van der Kruit and G. Gilmore, editors. IAU Symposium, Stellar Populations, Kluwer, Dordrecht, p. 85, 1995.

W. E. Harris and S. van den Bergh. AJ, 86:1627. 1981.

G. L. H. Harris, D. Geisler, H. C. Harris, and J. C. Hesser. AJ, 104:613, 1992.

W. E. Harris and R. E. Pudritz. ApJ, 429:177, 1994.

W. E. Harris, C. J. Pritchet, and R. D. McClure. ApJ, 441:120, 1995.

W. E. Harris, G. L. H. Harris, and D. E. McLaughlin. AJ, 115:1801, 1998.

M. Hilker, L. Infante, and T. Richtler. $A \mathscr{E} A S$, 138:55, 1999.

L. C. Ho. In J. Franco, R. Terlevich, and G. Tenorio-Tagle, editors, Starburst Activity in Galaxies, Reviews of Mex. Astron. Ap., 6:5, 1997.

J. A. Holtzman, S. M. Faber, E. J. Shaya, T. R. Lauer, J. Grothe, D. A. Hunter, W. A. Baum, S. P. Ewald, J. F. Hester, R. M. Light, C. R. Lynds, E. J. O’Neil, Jr., and J. A. Westphal. AJ, 103: 691, 1992.

J. P. Huchra and J. P. Brodie. AJ, 93:779, 1987.

N. Kaiser. ApJ, 284:L9, 1984.

D. Kaisler, W. E. Harris, D. R. Crabtree, and H. B. Richer. AJ, 111:2224, 1996.

M. Kissler-Patig. A\&A, 319:83, 1997.

M. Kissler-Patig, S. Kohle, M. Hilker, T. Richtler, L. Infante, and H. Quintana. A $E A, 319: 470,1997$.

M. Kissler-Patig, J. P. Brodie, L. L. Schroder, D. A. Forbes, C. J. Grillmair, and J. P. Huchra. AJ, 115:105, 1998a.

M. Kissler-Patig, D. A. Forbes, and D. Minniti. MNRAS, 298:1123, 1998b.

M. Kissler-Patig, C.J. Grillmair, G. Meylan, J. P. Brodie, D. Minniti, and P. Goudfrooij. AJ, 117:1206, 1999. 
Y. Kumai, Y. Hashi, and M. Fujimoto. ApJ, 416:576, 1993a.

Y. Kumai, B. Basu, and M. Fujimoto. ApJ, 404:144, 1993b.

A. Kundu and B. C. Whitmore. AJ, 116:2841, 1998.

A. Kundu, B. C. Whitmore, W. B. Sparks, F. D. Macchetto, F.D., S. E. Zepf, and K. M. Ashman. ApJ, 513:733, 1999.

S. S. Larsen and T. Richtler. A\&SA, submitted, 1999.

M. G. Lee and D. Geisler. AJ, 106:493, 1993.

D. E. McLaughlin. $A J$, 117:2398, 1999.

D. E. McLaughlin, W. E. Harris, and D. A. Hanes. ApJ, 422:486, 1994.

R. H. Mendez, M. A. Guerrero, K. C. Freeman, M. Arnaboldi, R. P. Kudritzki, U. Hopp, M. Capaccioli, and H. Ford. ApJ, 491:L23, 1997.

D. Merritt. In J.M. Dickey, editor, Minnesota Lectures on Clusters of Galaxies and Large-Scale Structure, ASP Conf. Ser., 5, p. 175, 1988.

G. R. Meurer, T. M. Heckman, C. Leitherer, A. Kinney, C. Robert, and D. R. Garnett. AJ, 110:2665, 1995.

B. W. Miller, B.C. Whitmore, F. Schweizer, and S. M. Fall. AJ, 114:2381, 1997.

B. W. Miller, J. M. Lotz, H. C. Ferguson, M. Stiavelli, and B. C. Whitmore. ApJ, 507:L133, 1998.

D. Minniti, G. Meylan, and M. Kissler-Patig. AEAA, 312:49, 1996.

D. Minniti, M. Kissler-Patig, P. Goudfrooij, and G. Meylan. AJ, 115:121, 1998.

J. R. Mould, J. B. Oke, and J. M. Nemec. AJ, 92:53, 1987.

J. R. Mould, J. B., Oke, P. T. de Zeeuw, and J. M. Nemec. AJ, 99:1823, 1990.

C. Murali and M. D. Weinberg. MNRAS, 288:767, 1997.

J. C. Muzzio. PASP, 99:245, 1987.

E. H. Neilson, Jr., Z. I. Tsvetanov, and H. C. Ford. ApJ, 483:745, 1997.

E. H. Neilson, Jr. and Z. I. Tsvetanov. ApJ, 515:L13, 1999.

P. Ostrov, D. Geisler, D., and J. C. Forte. AJ, 105:1762, 1993.

[] M. A. Pahre, S. G. Djorgovski, and R. R. de Carvalho. ApJ, 453:L15, 1995.

H. B. Richer, D. R. Crabtree, A. C. Fabian D. N. C. Lin. AJ, 105:877, 1993.

F. Schweizer. In S.M. Faber, editor, Nearly Normal Galaxies, Springer, New York, p. 18,1987

F. Schweizer, B. W. Miller, B. C. Whitmore, and S. M. Fall. AJ, 112:1839, 1996.

F. Schweizer. In M. Arnaboldi, G. S. Da Costa, and P. Saha, editors, The Nature of Elliptical Galaxies, ASP Conference Ser. 116, Astron.Soc.Pacific, San Francisco, p. 504, 1997.

J. Secker, D. Geisler, D. E. McLaughlin, and W. E. Harris. AJ, 109:1019, 1995.

T. Theuns, and S. J. Warren. MNRAS, 284:L11, 1997.

T. X. Thuan, and J. Kormendy. PASP, 89:466, 1977.

A. Toomre. In B.M. Tinsley and R.B. Larson, editors, The Evolution of Galaxies and Stellar Populations, Yale University Observatory, New Haven, p. 420, 1977.

A. van den Bergh. Vistas Astron., 21:71, 1977.

S. van den Bergh. PASP, 96:329, 1984.

S. van den Bergh. $A J, 108: 2145,1994$.

S. van den Bergh. ApJ, 450:27, 1995a

S. van den Bergh. $A J, 110: 2700,1995 \mathrm{~b}$

S. van den Bergh and W. E. Harris. AJ, 87:494, 1982.

R. P. van der Marel. MNRAS, 253:710, 1991.

M. L. Weil, J. Bland-Hawthorn, and D. Malin. ApJ, 490:664, 1997.

M. J. West. MNRAS, 265:755, 1993.

M. J. West, P. Cote, C. Jones, W. Forman, W., and R. O. Marzke. ApJ, 453:L77, 1995. 
B. C. Whitmore, F. Schweizer, C. Leitherer, K. Borne, and C. Roberts. AJ, 106:1354, 1993.

B. C. Whitmore and F. Schweizer. AJ, 109:960, 1995.

S. E. Zepf and K. M. Ashman. MNRAS, 264:611, 1993.

S. E. Zepf, D. Geisler, and K. M. Ashman. ApJ, 435:L117, 1994.

S. E. Zepf, K. M. Ashman, and D. Geisler. ApJ, 443:570, 1995. 
elmegreen.tex; 9/10/2018; 13:48; p.20 